\title{
Non-standardised treatment rooms present an obstacle to efficient and prompt patient care
}

\author{
Authors: Ruth Tunney, Samuel Shillito and Emma Wheatley
}

\section{Aims}

This project intended to determine whether the current layout of treatment rooms is conducive to the straightforward location of equipment, by analysing whether foundation year one (FY1) doctors were able to quickly and successfully perform routine tasks on a range of unfamiliar wards.

\section{Methods}

An initial survey was distributed to FY1 doctors to analyse their experiences of collecting equipment for venepuncture, cannulation and arterial blood gas sampling (ABG) on unfamiliar wards. FY1 volunteers were observed and timed while collecting equipment for these procedures on three wards with which they were unfamiliar. Prior to being observed, doctors were asked to estimate a reasonable task completion time. Doctors then rated the ease of equipment location and were invited to offer comments and suggestions for improvement.

\section{Results}

19 FY1 doctors participated in the initial survey. $89 \%$ indicated that the organisation of treatment rooms was inconsistent, and $79 \%$ could not collect equipment quickly or easily on an unfamiliar ward. Overall, 95\% said that the treatment rooms needed to be improved. When questioned about a colour-coded system introduced in $2009,90 \%$ of doctors were either unaware of this or did not understand it.

18 FY1 doctors volunteered and performed 54 individual tasks. The mean rating for ease of equipment location was 5.6/10. Overall, actual task completion times vs estimated times were $73 \%$ longer for venepuncture, $110 \%$ longer for cannulation and $143 \%$ longer for ABG. $50 \%$ of volunteers said that the ABG kit was the hardest item to find.

Frequent reference was made to inconsistent room layout, incorrect labelling and haphazard equipment storage. If a system was perceived, it was at times incorrectly understood. Suggestions for improvement included standardisation (67\%), equipment trolleys (50\%), clearer labelling (39\%) and colour coding (33\%).

Authors: Royal Bolton NHS Foundation Trust, Bolton, Lancashire, UK

\section{Conclusions}

This project uncovered widespread dissatisfaction regarding treatment rooms and demonstrated that an existing system was no longer maintained, causing doctors to spend valuable time searching for routine equipment. The potential delay of patient care raised an important patient safety issue. Standardisation has been successfully employed within the trust for the resuscitation trolleys used in cardiac arrests, and medical staff expressed a preference that this logic be applied to daily ward tasks.

These findings were presented to the Trust Clinical Governance Committee in June 2014, prompting the trust-wide reintroduction of the original system, which grouped equipment by function using colour coding and clear labelling. Clinical staff were educated in the use of this system using an education campaign.

\section{Conflict of interest statement}

None declared. 